\title{
Recurrent Apnea Following Occipital Encephalocele Surgery in a Child -Responding to Caffeine Citrate
}

\author{
Prerna Chaudhary, ${ }^{1}$ Amitabh Singh,${ }^{1,}{ }^{*}$ Rahul Jain, ${ }^{1}$ and Anirban Mandal ${ }^{2}$ \\ ${ }^{1}$ Department of Pediatrics, Chacha Nehru Bal Chikitsalaya, New Delhi, India \\ ${ }^{2}$ Department of Pediatrics, Sitaram Bhartia Institute of Science and Research, New Delhi, India \\ "Corresponding author: Amitabh Singh, Assistant Professor, Department of Pediatrics, Chacha Nehru Bal Chikitsalaya, New Delhi, 110031, India. Tel: +91-118447594629, E-mail: \\ dramit_amy@yahoo.co.in
}

Received 2016 October 02; Revised 2017 February 15; Accepted 2017 February 27.

\begin{abstract}
Introduction: Apnea denotes temporary cessation of breathing. It is an ominous symptom in children beyond neonatal period. Central apnea results from a lack of respiratory effort due to either a cessation of output from the brainstem respiratory centers or the inability of the efferent nerves and respiratory muscles to receive or process the signals from the brain. This can be due to the immaturity of the system as seen in premature infants or secondary to different disease processes.

Case Presentation: A 13-month-old girl child presented with frequent apneic spells following a major neurosurgical intervention for occipital encephalocele. Marked improvement was seen after the institution of caffeine citrate therapy resulting in complete resolution of apnea along with appreciable growth and development.

Conclusions: Recurrent apnea is a potential complication in infants following the repair of occipital encephalocele. Caffeine citrate may be effective in central apnea beyond the neonatal period especially in the treatment of apnea related to neurosurgical procedures.
\end{abstract}

Keywords: Neurosurgery, Central Apnea, Central Nervous System Stimulants

\section{Introduction}

The term 'apnea' denotes cessation of respiratory airflow. American academy of pediatrics (AAP) defines apnea in an infant as "an unexplained episode of cessation of breathing for 20 seconds or longer, or a shorter respiratory pause associated with bradycardia, cyanosis, pallor, and/or marked hypotonia (1). Apnea can be central, obstructive, or mixed. Central apnea results from a lack of respiratory effort due to either a cessation of output from the brainstem respiratory centers or the inability of the efferent nerves and respiratory muscles to receive or process the signals from the brain. This can be due to the immaturity of the system as seen in premature infants, or secondary to different disease processes (2).

Caffeine, a methylxanthine, is a central nervous system stimulant. Its citrate salt has been the drug of choice in the prevention and treatment of apnea in preterm newborns and has appropriately been described as a "silver bullet" in neonatology (3). Although, the exact mechanism of action of caffeine citrate is not known, several theories exist which have been attributed to mainly antagonism of adenosine receptors. These include a) stimulation of medullary respiratory center; b) increase in carbon dioxide sensitivity; c) induced bronchodilatation; d) increased diaphragmatic contractility leading to increased minute ventilation; e) improved respiratory pattern; and f) reduced hypoxic respiratory depression (4). Therefore, theoretically it is expected to be effective in other causes of central apnea apart from apnea of prematurity as well.

We report a 13-month-old girl child with recurrent apnea post neurosurgery for occipital encephalocele treated with caffeine citrate. We wanted to report this case as recurrent central apnea following surgery for occipital encephalocele is very rare and to the best of our knowledge, successful use of caffeine in apnea following neurosurgical procedures in children beyond neonatal period has not been documented yet.

\section{Case Presentation}

A 13-month-old girl child was admitted to our hospital for recurrent episodes of apnea and one episode of hypoxic seizures followed by unresponsiveness.

The child was the first product of a nonconsanguineous marriage, born full term (39 weeks and 3 days of gestational age) by normal vaginal institutional delivery and cried immediately after birth. Her birth weight was $2.75 \mathrm{~kg}$. She was found to have a sub-occipital encephalocele with intact sac at birth and work-up for other congenital anomalies was negative. There was no history of apnea or any other significant illness during 
infancy. At 9 months of age, she underwent an elective excision of sac and dural repair for primary defect and placement of VP shunt for prevention of hydrocephalus. Following the procedure, she developed recurrent apneic episodes requiring tactile stimulation but remained otherwise asymptomatic and was discharged.

Thereafter, she was lost to follow up and at presentation to our institute at 13 months of age, she was found to be in hypotensive shock following a hypoxic spell with severe failure to thrive. There was no history of fever, cough, chest retraction, difficulty in breathing, seizure, vomiting, jaundice, or abdominal distention. The parents gave history of recurrent episodes of apneas requiring tactile stimulation after discharge but medical attention was not sought for the same. She was admitted with a presumptive diagnosis of shock possibly secondary to prolonged hypoxia. There was initial improvement following fluid boluses, oxygen therapy, and inotropic support (Dopamine). Her hemodynamic status stabilized over next 48 hours and she could be weaned off oxygen as well. But, she continued to have frequent and severe apneic spells, requiring tactile stimulation. Various causes of secondary apnea were ruled out in the child (Table 1). There was no history of any medication intake by the child or her mother. NCCT (Non contrast computed tomography) of brain revealed VP (Ventriculo-peritoneal) shunt in situ in right frontal lobe parenchyma with hypoplastic cerebellar hemispheres and stretched brainstem and no evidence of raised intracranial pressure/ cerebral edema. Therefore, considering a diagnosis of apnea secondary to central nervous system dysfunction, she was started on caffeine citrate after discussion with her parents. It was started at a loading dose of 20 $\mathrm{mg} / \mathrm{kg}$ intravenously (IV) followed by a maintenance dose of $5 \mathrm{mg} / \mathrm{kg} 24$ hourly as IV infusion. She was monitored closely for adverse effects of caffeine therapy (e.g. tachycardia, irritability, feed intolerance, etc.) as facilities for therapeutic drug monitoring was not available. Only mild and transient tachycardia was observed during and in the immediate post-infusion period. Subsequently, the apneic spells decreased substantially and caffeine was made oral over next 4 days. Nutritional rehabilitation was started with the help of dietician and she could be discharged on oral caffeine citrate at a dose of $6 \mathrm{mg} / \mathrm{kg} /$ day after 2 weeks of hospitalization.

She was followed up closely with multidisciplinary approach involving pediatrician, neurosurgeon, dietician, and social service officer. Caffeine could be stopped after 1 month, as she remained completely apnea free following discharge. Over the next 6 months, she gained $2 \mathrm{~kg}$ of weight and also gained new developmental milestones with current developmental age of 9 months.

\section{Discussion}

Apnea is an ominous symptom in children beyond neonatal period. Our child presented with recurrent apneas following surgery for occipital encephalocele. A diagnosis of central apnea due to central nervous system dysfunction was made after ruling out various secondary causes of apnea and there was marked improvement with caffeine therapy.

Apnea following neurosurgical procedures has been well described in children but they are mostly either in the immediate post-operative period, post brain tumor surgery or are caused by obstructive apnea due to airway malformations (5). Obstructive sleep apnea (OSA) has been reported as a presenting feature of fronto-ethmoidal encephalocele (6), trans-alar, sphenoidal encephalocele (7) and also endoscopic surgical repair of encephalocele causing CSF rhinorrhea (8). Agarwal el al. have reported central apnea in a child with TectoCerebellar Dysraphia manifesting as occipital meningocoele (9). But central apnea following repair of occipital encephalocele has been sparingly reported in literature. Arnold Chiari syndrome (ACS) and other cranio-vertibral junction malformations are known to be associated with both central and obstructive apneas. The possible mechanisms are thought to be: 1. direct compression of the brain stem control centers for automatic breathing; 2 . compression of cranial nerves and 3. altered nerve pathways due to a syrinx (10). Though our patient did not have any radiological features suggestive of ACM, there was stretching of the brainstem which could have lead to a similar patho-physiological scenario.

Caffeine has been the panacea in the management of apnea of prematurity (3). In addition, in preterm, caffeine has established benefits in facilitating extubation, shortening the duration of intubation and noninvasive respiratory support, reduction in the incidence of bronchopulmonary dysplasia, decreasing need for treatment of patent ductus arteriosus, improving neuro-developmental outcome and reducing the severity of retinopathy of prematurity. Possible or apparent benefits of caffeine include prevention of intermittent hypoxia, postoperative apnea in preterm infants undergoing general anesthesia, apnea associated with bronchiolitis in young infants and induction of anti-inflammatory cytokine profile (4). Razak et al. (11) have also reported successful treatment of idiopathic/ primary apnea in a term infant with caffeine citrate. Use of caffeine benefitted adult patients with OSA undergoing uvulopalatopharyngoplasty as well (12).

Thus the use of caffeine in our patient was based on anecdotal evidence and an understanding of its clinical efficacy coupled with indispensability of the situation. But the child improved quite markedly, suggesting perhaps 
Table 1. Investigations Performed to Rule Out Secondary Causes of Apnea in the Index Child

\begin{tabular}{|c|c|}
\hline Potential Cause of Apnea & Relevant Investigation \\
\hline Sepsis & Negative sepsis screen; sterile blood and urine culture \\
\hline Meningitis & Normal CSF cytology, biochemistry and sterile bacterial and fungal culture \\
\hline Seizure & Normal EEG \\
\hline Congenital heart disease & Normal echocardiography \\
\hline Pulmonary disease & Normal chest $\mathrm{x}$-ray and normal arterial blood gases in interval period \\
\hline Gastroesophageal reflux disease & No evidence of reflux on gastroesophageal reflux (GER) scan and barium swallow \\
\hline Upper airway anomaly & Normal fiber-optic laryngoscopy \\
\hline Metabolic disturbances (Hypoglycemia, Hypocalcemia, Hyponatremia) & $\begin{array}{l}\text { Normal blood sugar, serum total and ionized calcium, serum sodium measured } \\
\text { multiple times }\end{array}$ \\
\hline Inborn error of metabolism & $\begin{array}{l}\text { Normal blood ammonia, serum lactate, Tandem mass spectrometry (TMS) and urinary } \\
\text { Gas chromatography and mass spectrometry (GCMS) }\end{array}$ \\
\hline
\end{tabular}

a possible new therapeutic indication of an established pharmacological agent used for management of apneas in preterm newborn.

\subsection{Conclusion}

Recurrent apnea is a potential complication in infants following repair of occipital encephalocele. Caffeine citrate may be effective in central apnea beyond the neonatal period especially in the treatment of apnea related to neurosurgical procedures.

\section{Acknowledgments}

Nil.

\section{Footnotes}

Authors' Contribution: All the authors were involved in the management of the case, literature review, and manuscript writing. All the authors approved the final manuscript.

Implications for Health Policy Makers/Practice/Research/Medical Education: Anticipation, early detection, and effective management of recurrent apnea following neurosurgeries in children.

Financial Disclosure: Nil.

Role of the sponsor: Nil.

\section{References}

1. Committee on F, Newborn. American Academy of P. Apnea, sudden infant death syndrome, and home monitoring. Pediatrics. 2003;111(4 Pt 1):914-7. doi: 10.1542/peds.111.4.914. [PubMed: 12671135].
2. Paolillo P, Picone S. Apnea of prematurity. J Pediatr and Neonatal Indiv Med (JPNIM). 2013;2(2).

3. Aranda JV, Beharry K, Valencia GB, Natarajan G, Davis J. Caffeine impact on neonatal morbidities. J Matern Fetal Neonatal Med. 2010;23 Suppl 3:20-3. doi: 10.3109/14767058.2010.517704.[PubMed: 20873976].

4. Dobson NR, Hunt CE. Pharmacology review: Caffeine use in neonates: Indications, pharmacokinetics, clinical effects, outcomes. Neo Rev. 2013;14(11):540-50. doi:10.1542/neo.14-11-e540.

5. Mekitarian Filho E, Carvalho WB, Cavalheiro S. Perioperative patient management in pediatric neurosurgery. Rev Assoc Med Bras (1992). 2012;58(3):388-96. doi: 10.1016/S0104-4230(12)70212-9. [PubMed: 22735234].

6. Murali R, Douyon P, Omeis I. Large anterior skull base lesions in patients presenting with obstructive sleep apnea. Report of two cases and review of the literature. J Neurosurg. 2004;101(1):163-6. doi: 10.3171/jns.2004.101.1.0163. [PubMed: 15255269].

7. Mylanus EA, Marres HA, Vlietman J, Kollee LA, Freihofer HP, Thijssen $\mathrm{HO}$, et al. Transalar sphenoidal encephalocele and respiratory distress in a neonate: a case report. Pediatrics. 1999;103(1):E12. doi: 10.1542/peds.103.1.e12. [PubMed: 9917492].

8. Fleischman GM, Ambrose EC, Rawal RB, Huang BY, Ebert CJ, Rodriguez $\mathrm{KD}$, et al. Obstructive sleep apnea in patients undergoing endoscopic surgical repair of cerebrospinal fluid rhinorrhea. Laryngoscope. 2014;124(11):2645-50. doi: 10.1002/lary.24661. [PubMed: 24591190].

9. Agrawal A, Joharapurkar SR, Khan AU. Tecto-cerebellar dysraphia manifesting as occipital meningocoele associated with congenital melanocytic nevi and pectus excavatum. Iran J Pediatr. 2010;20(1):11822. [PubMed: 23056693].

10. Botelho RV, Bittencourt LR, Rotta JM, Tufik S. A prospective controlled study of sleep respiratory events in patients with craniovertebral junction malformation. J Neurosurg. 2003;99(6):1004-9. doi: 10.3171/jns.2003.99.6.1004. [PubMed: 14705728].

11. Razak A, Nagesh NK. Caffeine in a term neonate with apnea. Indian Pediatr. 2015;52(4):346-7. [PubMed: 25929643].

12. Gouda NM. Intravenous caffeine for adult patients with obstructive sleep apnea undergoing uvulopalatopharyngoplasty: Effects on postoperative respiratory complications and recovery profile. Med J Cairo Univ. 2010;78(2):155-9. 